\title{
ENTIRE FUNCTIONS WITH ALMOST RADIALLY DISTRIBUTED VALUES
}

\author{
SHIGERU KIMURA
}

\begin{abstract}
Let $f(z)$ be an entire function of finite lower order. Assume that there exist a positive number $h$ and an unbounded sequence $\left\{w_{n}\right\}_{n=1}^{\infty}$ such that all roots of the equations $f(z)=w_{n}(n=1,2, \ldots)$ lie in $\{z ;|\operatorname{Im} z|<$ $h$ \}. Then $f(z)$ is a polynomial of degree not greater than two. The hypothesis of the finiteness of lower order of $f(z)$ cannot be removed.
\end{abstract}

1. Edrei [2] studied meromorphic functions with three radially distributed values and he proved the following elegant theorem.

THEOREM A. Let $f(z)$ be an entire function. Assume that there exists an unbounded sequence $\left\{w_{n}\right\}_{n=1}^{\infty}$ such that all the roots of the equations $f(z)=w_{n}$ $(n=1,2, \ldots)$ be real. Then $f(z)$ is a polynomial of degree not greater than two.

We extend Theorem $A$ and show the following.

THEOREM. Let $f(z)$ be an entire function of finite lower order. Assume that there exist a positive number $h$ and an unbounded sequence $\left\{w_{n}\right\}_{n=1}^{\infty}$ such that all roots of the equations $f(z)=w_{n}(n=1,2, \ldots)$ lie in $\{z ;|\operatorname{Im} z|<h\}$. Then $f(z)$ is a polynomial of degree not greater than two.

We note that the hypothesis of the finiteness of the lower order of $f(z)$ cannot be removed from our Theorem. In fact, Fuchs and Hayman [4, p. 81] proved that there exists an entire function $f(z)$, such that in the strip $A=\{z=x+i y ; x>0,|y| \leqslant \pi\} \quad f(z)=\exp \left(e^{z}+z\right)+O\left(z^{-2}\right)$, while outside $A, f(z)=O\left(z^{-2}\right)$ uniformly as $z \rightarrow \infty$.

The proof of the Theorem goes in two stages: (1) $f(z)$ is an entire function of order at most one, (2) $f(z)$ is a polynomial. The proof of (1) follows closely the corresponding steps in the proof of Theorem 1 in [1]. The proof of (2) is quite different from that of Edrei.

The author wishes to express his thanks to the referee for his valuable advice.

Received by the editors March 26, 1977 and, in revised form, September 6, 1977.

AMS (MOS) subject classifications (1970). Primary 30A70.

Key words and phrases. Entire function, almost radially distributed value, lower order, deficiency. 
2. Lemmas. Our starting point will be the following three lemmas obtained by Edrei.

Consider the $q$ radii defined by

$$
r e^{i \omega_{1}}, \ldots, r e^{i \omega_{q}} \quad(r \geqslant 0)
$$

where $0 \leqslant \omega_{1}<\cdots<\omega_{q}<2 \pi(q \geqslant 1)$.

LEMMA A [1]. Let $\Delta_{k}(\delta)$ be the sector defined by

$$
r>1, \quad \omega_{k}+\delta<\theta<\omega_{k+1}-\delta \quad(k=1, \ldots, q),\left[\omega_{q+1}=2 \pi+\omega_{1}\right]
$$

and write $\pi / \gamma=\omega_{k+1}-\omega_{k}-2 \delta$. Consider the conformal transformation

$$
w=\frac{u^{\gamma}-u^{-\gamma}-\tau}{u^{\gamma}-u^{-\gamma}+\tau}=\phi_{k}^{-1}(u)
$$

where $\tau$ is a positive parameter and $u=e^{-i \zeta_{k}} z, \zeta_{\kappa}=\left(\omega_{k}+\omega_{k+1}\right) / 2$. Then the function $z=e^{i \zeta_{k}} \phi_{k}(w)$ maps the circle $|w|<1$ onto the sector $\Delta_{k}(\delta)$, and we have

$$
\tau / 4 r^{-\gamma} \cos \left(\gamma\left[\theta-\zeta_{k}\right]\right)<1-|w|<8 \tau r^{-\gamma} \quad(|z|=r) .
$$

The following Lemma $B$ is a modification of Lemma 1 in [1].

LEMMA B. Let $f(z)$ be meromorphic in the region $\{z ; 1<|z|<+\infty\}$ and assume that for any $\delta>0$ all but a finite number of the roots of the three equations $f(z)=0, f(z)=\infty, f^{(m)}(z)=1\left(m \geqslant 0, f^{(0)}=f\right)$ lie in the angles $\left|\arg z-\omega_{k}\right|<\delta(k=1, \ldots, q)$. Consider the $q$ functions defined by $F_{k}(w)=$ $f\left(e^{i \xi_{k}} \phi_{k}(w)\right)(k=1, \ldots, q)$. Then as $t \rightarrow 1(0<t<1)$

$$
\begin{gathered}
m\left(t, F_{k}(w)\right)=O\left(\log \frac{1}{1-t}\right), \quad m\left(t, F_{k}(w)^{-1}\right)=O\left(\log \frac{1}{1-t}\right), \\
m\left(t, \frac{1}{f^{(m)}\left(e^{i \zeta_{k}} \cdot \phi_{k}(w)\right)-1}\right)=O\left(\log \frac{1}{1-t}\right) .
\end{gathered}
$$

LEMMA C [2]. Let $G(t)$ be a positive, real, continuous and nondecreasing function defined for $t \geqslant t_{0}>0$. Assume that the order $\rho$ and the lower order $\mu$ of $G(z)$ satisfy $\mu<\rho(\rho \leqslant+\infty)$ and let $\sigma, \tau$ be given such that $\mu<\sigma<\tau<$ $\rho$. Then there exist arbitrary large values of $r$ such that

$$
\frac{G(r)}{r^{\tau}} \geqslant \frac{G(t)}{t^{\tau}} \quad\left(t_{0} \leqslant t \leqslant r^{\tau / \sigma}\right), \quad \frac{G(r)}{r^{\tau}} \geqslant 1 .
$$

LEMMA D. Let $h(z)(\neq$ constant) be a meromorphic function of finite lower order $\mu$, order $\rho(\leqslant+\infty)$, the poles of which have a positive deficiency. Then there exists a sequence of Pólya peaks $\left\{r_{n}\right\}$ of finite order $\tau$ of $T(r, h)$ such that $\mu \leqslant \tau \leqslant \rho$ and

$$
\tau \leqslant \liminf _{n \rightarrow \infty} \frac{\log T\left(r_{n}, h\right)}{\log r_{n}},
$$

and further there exist two positive numbers $K$ and $A$ such that

$$
\text { measure } J\left(r_{n}\right)>A
$$


for all sufficiently large $n$ where

$$
J\left(r_{n}\right)=\left\{\theta ; 0 \leqslant \theta<2 \pi,\left|h\left(r_{n} e^{i \theta}\right)\right|>\exp \left[K \cdot T\left(r_{n}, h\right)\right]\right\} .
$$

Proof. We can define a sequence of Pólya peaks $\left\{r_{n}\right\}$ of finite order $\tau$ $(\mu \leqslant \tau \leqslant \rho)$ satisfying (2.1) in view of Lemma $C$ by a routine argument [6]. Now we shall prove that the sequence satisfies (2.2). If (2.2) were false, then there would exist sequences $\varepsilon_{n} \rightarrow 0, \lambda_{n} \rightarrow 0$ such that for infinitely many $n$

$$
\text { measure }\left\{\theta ; 0 \leqslant \theta<2 \pi,\left|h\left(r_{n} e^{i \theta}\right)\right|>\exp \left[\varepsilon_{n} T\left(r_{n}, h\right)\right]\right\}<\lambda_{n} .
$$

Using a result of Edrei and Fuchs [3, p. 322], we obtain

$$
m\left(r_{n}, h\right) \leqslant \varepsilon_{n} T\left(r_{n}, h\right)+22 T\left(2 r_{n}, h\right) \lambda_{n}\left\{1+\log ^{+} 1 / \lambda_{n}\right\} .
$$

Since $\left\{r_{n}\right\}$ is a sequence of Pólya peaks, we have $m\left(r_{n}, h\right)=o\left(T\left(r_{n}, h\right)\right)$ $(n \rightarrow \infty)$ which conflicts with the fact that the poles of $h(z)$ have a positive deficiency. Thus we have proved the lemma.

Using the notations of Lemma A, we prove the following.

LEMMA E. Let $h(z)$ be a meromorphic function of finite lower order $\mu$ and of order $\rho$ (not necessarily finite). Assume that for any $\delta>0$ all but a finite number of the poles of $h(z)$ lie in the angles $\left\{z ;\left|\arg z-\omega_{k}\right|<\delta\right\}(k=$ $1, \ldots, q)$ and that the poles of $h(z)$ have a positive deficiency. Then

$$
\rho>\beta=\sup \left\{\frac{\pi}{\omega_{2}-\omega_{1}}, \ldots, \frac{\pi}{\omega_{q+1}-\omega_{q}}\right\}
$$

implies

$$
\limsup _{t \rightarrow 1} \frac{m\left(t, h\left(e^{i \zeta_{k}} \phi_{k}(w)\right)\right)}{\log 1 /(1-t)}=+\infty \quad(0<t<1),
$$

for some integer $k(1 \leqslant k \leqslant q)$.

Proof. Choose $\delta$ sufficiently small such that $\delta<A / 2 q$ where $A$ is the positive number defined in Lemma $\mathrm{D}$, and such that

$$
\rho \geqslant \rho^{\prime}>\gamma^{\prime}+\eta, \quad \rho^{\prime} \geqslant \mu, \quad \rho^{\prime}<+\infty
$$

in view of the assumption $\beta<\rho$ where

$$
\gamma^{\prime}=\sup \left\{\frac{\pi}{\omega_{2}-\omega_{1}-2 \delta}, \ldots, \frac{\pi}{\omega_{q+1}-\omega_{q}-2 \delta}\right\}
$$

and $\eta$ is a positive number. If we choose a sequence of Pólya peaks $\left\{r_{n}\right\}$ of order $\rho^{\prime}$ of $h(z)$ defined in Lemma D satisfying

$$
\liminf _{n \rightarrow \infty} \frac{\log T\left(r_{n}, h\right)}{\log r_{n}} \geqslant \rho^{\prime},
$$

then in view of (2.2) we may associate with each $r_{n}\left(n \geqslant n_{0}\right)$ at least one argument $\theta_{n}$ belonging to one of the $q$ arcs defined by $\omega_{k}+A / 2 q \leqslant \theta \leqslant$ $\omega_{k+1}-A / 2 q$ and such that

$$
\log \left|h\left(r_{n} e^{i \theta} n\right)\right|>K \cdot T\left(r_{n}, h\right) .
$$


We choose among the $q$ sectors $\Delta_{1}(\delta), \ldots, \Delta_{q}(\delta)$ which are defined in Lemma $A$, a sector $\Delta_{k}(\delta)$ which contains an infinity of terms of $r_{1} e^{i \theta_{1}}$, $r_{2} e^{i \theta_{2}}, \ldots$ Renumbering if necessary the elements of this sequence, we may assume that all its terms belong to $\Delta_{k}(\delta)$. Hence, using the notations of Lemma A, we obtain

$$
\cos \gamma\left[\theta_{n}-\zeta_{k}\right]>\cos (\pi / 2-A \gamma / 2 q+\delta \gamma) \quad(>0) .
$$

The function $H_{k}(w)=h\left(e^{i \zeta_{k}} \phi_{k}(w)\right)$ is regular in the unit circle except for a finite number of points. If we put $w_{n}=\phi_{k}^{-1}\left(r_{n} e^{i\left(\theta_{n}-\zeta_{k}\right)}\right),(2.6)$ becomes

$$
\log \left|H_{k}\left(w_{n}\right)\right|>K \cdot T\left(r_{n}, h\right) \text {. }
$$

In view of (2.7) and the inequality of Lemma $A$ we obtain

$$
\frac{B}{r_{n}^{\gamma}}=\frac{\cos (\pi / 2-A \gamma / 2 q+\delta \gamma)}{4 r_{n}^{\gamma}}<1-\left|w_{n}\right| .
$$

Taking $t_{n}=\left|w_{n}\right|+\left(1-\left|w_{n}\right|\right) / 2$, we have

$$
\begin{aligned}
& m\left(t_{n}, H_{k}(w)\right)+O\left(\log \frac{1}{1-t_{n}}\right) \\
& \geqslant \frac{t_{n}-\left|w_{n}\right|}{t_{n}+\left|w_{n}\right|} \log \left|H_{k}\left(w_{n}\right)\right| \quad\left(n \geqslant n_{0}\right),
\end{aligned}
$$

so that (2.8), (2.9) and (2.10) imply

$$
m\left(t_{n}, H_{k}(w)\right)+O\left(\log \frac{1}{1-t_{n}}\right) \geqslant \frac{B K}{4 r_{n}^{\gamma}} T\left(r_{n}\right) .
$$

If the lemma were not true, then we would have

$$
m\left(t, H_{k}(w)\right)=O\left(\log \frac{1}{1-t}\right) .
$$

Combining (2.11) and (2.12), we obtain

$$
K^{\prime} \log \frac{r_{n}^{\gamma}}{B} \geqslant \frac{B K}{4} \frac{T\left(r_{n}\right)}{r_{n}^{\gamma}}
$$

where $K^{\prime}$ is a positive number, and hence $T\left(r_{n}\right)<r_{n}^{\gamma+\eta}$ provided $n$ is large enough. Thus we have $\rho^{\prime} \leqslant \gamma+\eta \leqslant \gamma^{\prime}+\eta$ by (2.5) which contradicts (2.4). This contradiction proves the lemma.

Using Lemma B and Lemma $\mathrm{E}$ we can prove the following Lemma 1, by the reasonings similar to those of Theorem 1 in [1].

LEMMA 1. Let $f(z)$ be a meromorphic function of finite lower order and such that for any $\delta>0$ all but a finite number of roots of the three equations $f(z)=0, f(z)=\infty, f^{(m)}(z)=1$ lie in the angles $\left\{z ;\left|\arg z-\omega_{k}\right|<\delta\right\}(k=$ $1, \ldots, q)$. Denote by $\delta\left(a, f^{(m)}\right)$ the deficiency of the value a of the function $f^{(m)}$ and assume

$$
\delta(0, f)+\delta\left(1, f^{(m)}\right)+\delta(\infty, f)>0 .
$$

Then the order $\rho$ of $f$ is necessarily finite and $\rho \leqslant \beta$. 
Further we quote the following lemma which is easily proved by a result of Heins [5, p. 70].

LEMMA F. Suppose that $u(z)$ is a subharmonic function in the finite plane which is not negative and not identically constant. If the following conditions are fulfilled for $r>r_{0}(\geqslant 0)$ : (1) $\inf _{|z|=r} u(z)=0$, (2) the angular measure of $\{u(z)=0,|z|=r\} \geqslant \phi_{0}(\geqslant 0)$. Then the order $\rho_{u}$ of $u(z)=$ $\lim \sup _{r \rightarrow \infty} \log \sigma(r) / \log r \geqslant \pi /\left(2 \pi-\phi_{0}\right)$ where $\sigma(r)=\sup _{|z|=r} u(z)$.

Using this lemma, we have

LEMMA 2. Suppose that an unbounded region $\Omega$ is contained in an angular region $\Delta=\{z ;|\arg z+\pi / 2|<\alpha\}$. Let $h(z)$ be an arbitrary positive harmonic function with boundary value zero in $\Delta$ and let $u(z)$ be an arbitrary positive harmonic function with boundary value zero in $\Omega$. Extending $h(z), u(z)$ to the whole plane as the subharmonic functions by the standard method and denoting them by the same notations, we have $\rho_{u} \geqslant \rho_{h}$.

Proof. By the Picard principle $h(z)$ is represented as

$$
h(z)=c r^{\pi / 2 \alpha} \cos (\theta+\pi / 2) \pi / 2 \alpha, \quad z=r e^{i \theta},
$$

where $c$ is a constant positive value. Hence $\rho_{h}=\pi / 2 \alpha$. Therefore $\rho_{u}$ is estimated as follows by Lemma $F$,

$$
\rho_{u} \geqslant \pi /\left(2 \pi-\phi_{0}\right) \geqslant \pi / 2 \alpha=\rho_{h} .
$$

3. Proof of the Theorem. The genus of $f(z)$ is not greater than one by Lemma 1. First we assume that the genus of $f(z)$ is one. Further we may assume that $w_{1}=0$ and $\left|w_{1}\right|<\left|w_{2}\right|<\cdots<\left|w_{n}\right| \rightarrow+\infty(n \rightarrow \infty)$. Then $f(z)$ may be represented as

$$
f(z)=\lambda e^{a z} \prod_{j=1}^{\infty}\left(1-\frac{z}{z_{j}}\right) \exp \left(\frac{z}{z_{j}}\right)
$$

where $\lambda(\neq 0)$ is a constant. Since the genus of $f(z)$ is one, putting $z_{j}=r_{j} e^{i \theta_{j}}$ we have

$$
\left|\sum_{j=1}^{\infty} \operatorname{Im} \frac{1}{z_{j}}\right| \leqslant \sum_{j=1}^{\infty} \frac{\left|r_{j} \sin \theta_{j}\right|}{r_{j}^{2}}<h \sum_{j=1}^{\infty} \frac{1}{r_{j}^{2}}<+\infty .
$$

Therefore $f(z)$ may be rewritten as

$$
f(z)=\lambda e^{b z} \prod_{j=1}^{\infty}\left(1-\frac{z}{z_{j}}\right) \exp \left\{\left(\operatorname{Re} \frac{1}{z_{j}}\right) z\right\}
$$

where $b=a+i \sum_{j=1}^{\infty} \operatorname{Im} 1 / z_{j}$. We may suppose $\operatorname{Im} b \geqslant 0$, otherwise we can replace $f(z)$ by $\overline{f(\bar{z})}$.

We consider

$$
\frac{z f^{\prime}(z)}{f(z)}=z\left\{\sum_{j=1}^{\infty}\left(\frac{1}{z-z_{j}}+\operatorname{Re} \frac{1}{z_{j}}\right)+b\right\}
$$


in $\{z ;|\arg z+\pi / 2|<\alpha<\pi / 2\}$. Let $K$ be a positive number such that $K \cdot \sin \alpha \geqslant 2 \pi$. Then there exists a positive number $r_{1}=r_{1}(K)$ such that

$$
\left|\frac{z \cdot f^{\prime}(z)}{f(z)}\right| \geqslant|z|\left|\operatorname{Im} \frac{f^{\prime}(z)}{f(z)}\right|>K
$$

in $\left\{z ;|\arg z+\pi / 2| \leqslant \alpha,|z|>r_{1}\right\}$.

Next if the genus of $f(z)$ is zero, then (3.1) is similarly proved.

Now we can easily show that $|f(-i y)| \rightarrow+\infty$ as $y \rightarrow+\infty$. We choose $w_{n}$ such that $|f(z)|<\left|w_{n}\right|$ for $|z| \leqslant r_{1}$. Let $\Omega$ be the component of $\{z ;|f(z)|>$ $\left.\left|w_{n}\right|\right\}$ which contains the negative imaginary axis from some point on. If $\Omega \subset\{z ;|\pi / 2+\arg z|<\alpha<\pi / 2\}$, then putting $u(z)=\log |f(z)|-\log \left|w_{n}\right|$ the order of $f(z)$ is greater than one by Lemma 2 which is impossible. Therefore we may assume that $\Omega \cap\{z ;|\pi / 2+\arg z| \leqslant \alpha\}$ contains an arc of a level curve $\gamma$ of $f(z)$ which joins a point of the ray $\arg z=-\pi / 2-\alpha$ to a point of the ray $\arg z=-\pi / 2$ and lies in $\left\{z ;|z|>r_{1}\right\}$. If the equation of $\gamma$ is given in parametric form $z=z(t)(0 \leqslant t \leqslant 1)$, then we have

$$
w=f(z(t)), \quad \frac{1}{w} \cdot \frac{d w}{d t}=\frac{z f^{\prime}(z)}{f(z)} \cdot \frac{z^{\prime}(t)}{z},
$$

and if we put $w=\left|w_{n}\right| e^{i \phi(t)}$, then we have

$$
\frac{d w}{d t}=i w \frac{d \phi}{d t} .
$$

Hence $|d \phi / d t|=\left|z f^{\prime}(z) / f(z)\right| \cdot\left|z^{\prime}(t) / z\right|$ and by (3.1) we have

$$
\int_{0}^{1}\left|\frac{d \phi}{d t}\right| d t \geqslant \frac{K}{r_{0}} \int_{0}^{1}\left|z^{\prime}(t)\right| d t \geqslant \frac{K}{r_{0}}\left(r_{0} \sin \alpha\right)=K \cdot \sin \alpha \geqslant 2 \pi
$$

where $r_{0}=\max _{0 \leqslant t \leqslant 1}|z(t)|$. In view of $f^{\prime}(z) \neq 0$ on $\gamma$, as $z$ traverses $\gamma$ in the fixed direction, $w$ traverses the circle $\Gamma ;|w|=\left|w_{n}\right|$ in the fixed direction and $\phi$ increases or decreases at least $K \cdot \sin \alpha$. Thus $w$ traverses the whole of $\Gamma$ and in particular $f(z)=w_{n}$ for some point $z \in \gamma$. But this contradicts the assumption of the Theorem. Hence if $f(z)$ satisfies the conditions of the Theorem, then $f(z)$ must be a polynomial. Then it is easy to show that the degree of $f(z)$ is at most two.

\section{REFERENCES}

1. A. Edrei, Meromorphic functions with three radially distributed values, Trans. Amer. Math. Soc. 78 (1955), 276-293.

2. _ The deficiencies of meromorphic functions of finite lower order, Duke Math. J. 31 (1964), 1-22.

3. A. Edrei and W. H. G. Fuchs, Bounds for the number of deficient values of certain classes of meromorphic functions, Proc. London Math. Soc. 12 (1962), 312-344.

4. W. K. Hayman, Meromorphic functions, Math. Monographs, Clarendon Press, Oxford, 1965.

5. M. Heins, On a notion of convexity connected with a method of Carleman, J. Analyse Math. 3 (1959), 53-77.

6. D. F. Shea, On the Valiron deficiencies of meromorphic functions of finite order, Trans. Amer. Math. Soc. 124 (1966), 201-227.

Department of Mathematics, Utsunomiya University, Mine-Machi Utsunomiya, Japan 